\title{
Aplicaçáo das Análises Biomecânicas da Pressáo Plantar na Marcha de Indivíduos com Doença de Parkinson
}

\author{
Application of Biomechanical Analysis of Plantar Pressure During Gait In Individuals with Parkinson's Disease
}

\author{
Luana Santos Gonçalves', Nathalie Ribeiro Artigas', \\ Carlos Roberto de Mello Rieder ${ }^{2}$
}

\begin{abstract}
RESUMO
Com o advento de novas tecnologias de pesquisas na área da biomecânica, pode-se obter dados sobre controle motor, coordenação e equilíbrio postural em várias condiçôes que provocam alterações na marcha, como ocorre na Doença de Parkinson (DP). Tais dados são de grande importância para evoluçóes nos tratamentos destes pacientes. Objetivo. Realizar uma revisão de literatura sobre análises biomecânicas da marcha em pacientes com DP, enfatizando aquelas relacionadas aos dados de distribuição da pressão plantar, buscando compreender a evolução das pesquisas nesta área e quais as perspectivas e necessidades de futuros estudos. Método. foram realizadas buscas nos sites dos Periódicos Capes, Pubmed e Scielo, selecionando estudos sobre análises quantitativas da marcha em pacientes com DP executados entre 1991 e 2012, sem restrição de idiomas. Resultados. Foram encontrados 1679 estudos, sendo 1654 excluídos devido aos critérios pré-determinados, totalizando 25 estudos incluídos. Conclusóes. Há concordância nas pesquisas quanto aos parâmetros espaciais e temporais da marcha na DP. Entretanto, o mesmo não ocorre com relação aos dados cinéticos. As análises de força e de pressão plantar ainda apresentam resultados controversos e metodologias questionáveis, sendo que novas pesquisas utilizando protocolos precisamente definidos poderão trazer benefícios ao tratamento dos pacientes com DP.
\end{abstract}

Unitermos. Biomecânica, Marcha, Doença de Parkinson.

Citação. Gonçalves LS, Artigas NR, Rieder CRM. Aplicação das Análises Biomecânicas da Pressáo Plantar na Marcha de Indivíduos com Doença de Parkinson.

\begin{abstract}
With the advent of new technologies researches in biomechanics, it is possible to obtain data in terms of motor control, coordination and postural balance in various conditions which cause changes in gait, as it occurs in Parkinson's Disease (PD). Such data have a great importance for the evolution of the treatments for these patients. Objective. a review of the literature concerning biomechanical analysis of gait in PD patients was realized, emphasizing these related to data distribution of plantar pressure in order to understand the evolution of researches in this area and what are the perspectives and needs of future studies. Method. the analysis was realized in the Capes Periodics, Pubmed and Scielo with the selection of studies in relation to quantitative analysis of gait in PD which were performed between 1991 and 2011, without language restriction. Results. 1679 studies were found and 1654 were excluded due to predetermined criteria, consequently, 25 studies were included. Conclusions. it seems to have a concordance in spatiotemporal parameters of gait in PD. However, the same is not true in relation to kinetics parameters. The force and plantar pressure analysis still showed controversial results and questionable methodologies, given that new researches using precisely defined protocols will be able to bring benefits to the treatment of patients with DP.
\end{abstract}

Keywords. Biomechanics, Gait, Parkinson Disease.

Citation. Gonçalves LS, Artigas NR, Rieder CRM. Application of Biomechanical Analysis of Plantar Pressure During Gait In Individuals with Parkinson's Disease.
Estudo realizado na Universidade Federal de Ciências da Saúde de Porto Alegre (UFCSPA), Porto Alegre-RS, Brasil.

1.Fisioterapeuta, Mestranda em Ciências da Reabilitação pela Universidade de Ciências da Saúde de Porto Alegre (UFCSPA), Porto Alegre-RS, Brasil.

2.Neurologista, Doutor em Clinical Neuroscience, Birmingham University England. Professor de Neurologia da Universidade de Ciências da Saúde de Porto Alegre (UFCSPA), Coordenador da Clínica de Parkinson do Hospital de Clinicas de Porto Alegre (HCPA), Porto Alegre-RS, Brasil.
Endereço para correspondência: Carlos RM Rieder Universidade Federal Ciências da Saúde de Porto Alegre, Departamento de Clínica Médica/Neurologia Avenida Osvaldo Aranha, 245 CEP 90050-170, Porto Alegre-RS, Brasil. E-mail: carlosrieder@terra.com.br 


\section{INTRODUÇÃO}

O distúrbio da marcha é um dos principais sintomas na Doença de Parkinson (DP), uma doença neurodegenerativa progressiva, que tem como principal base das manifestaçóes motoras a depleção de dopamina no estriado em decorrência da degeneração das aferências da substância nigra ${ }^{1}$.

O advento de instrumentos de análises da marcha, como plataformas de força e plataformas de pressão, dentre outros, tem aprimorado a obtenção de informaçóes cada vez mais precisas acerca da marcha humana e suas alteraçóes. Nesse sentido, pesquisadores ${ }^{2-6}$ vêm investigando alteraçôes biomecânicas na marcha dos indivíduos que sofrem da DP. Muitos desses estudos ${ }^{5,7}$ correlacionam as alterações da marcha com o grau de severidade da doença segundo escalas de referência. Em outros ${ }^{8,9}$, há correlação com o tratamento medicamentoso e até mesmo cirúrgico $^{10-12}$, possibilitando a quantificação de dados tanto cinéticos quanto cinemáticos, sendo os últimos mais ricamente investigados nos últimos anos.

A avaliação da marcha é, frequentemente, feita através da interpretação observacional, a qual pode variar bastante dependendo do avaliador. Por isso, técnicas de análise quantitativa de marchas patológicas, como a parkinsoniana, vêm sendo desenvolvidas para fornecer medidas objetivas ${ }^{13}$. Em geral, pacientes nos estágios mais avançados da DP possuem uma tendência a pisar simultaneamente com o calcanhar e o antepé no chão, resultando em um grave pé plano (flat foot strike), podendo, até mesmo, ocorrer o contato dos dedos no chão antes do calcanhar ${ }^{14}$.

O objetivo do presente estudo foi realizar uma revisão de literatura sobre análises biomecânicas da marcha de indivíduos com DP, enfatizando àquelas relacionadas aos dados de distribuição da pressão plantar nestas pessoas. Busca-se investigar a aplicação das pesquisas nesta área e quais as perspectivas e necessidades de futuros estudos.

\section{MÉTODO}

Para a elaboração desta revisão de literatura, foram consultadas as bases eletrônicas PubMed, Scielo e portal de Periódicos Capes, no período entre junho e novembro de 2011. Foram inclusos estudos publicados no período de 1991 a 2011, sem restrição de idioma, identificados através dos descritores: biomecânica, marcha, doença de Parkinson e seus correlatos em inglês.

Os critérios de inclusão adotados foram: artigos referentes à marcha de idosos saudáveis e idosos com DP, artigos que avaliam a marcha em indivíduos com DP através de instrumentos de análise quantitativa (cinética e cinematicamente), artigos referentes aos efeitos das medicaçôes dopaminérgicas na DP sobre a marcha e comportamento motor, e artigos referentes a intervençóes não-medicamentosas na $\mathrm{DP}$ e suas consequências sobre a marcha e comportamento motor.

Foram excluídos artigos que não realizassem análises quantitativas da marcha humana em idosos e indivíduos com DP.

\section{RESULTADOS E DISCUSSÃO}

Através da revisão realizada foram encontrados 1679 estudos, sendo 1654 excluídos devido aos critérios pré-determinados, totalizando 25 estudos incluídos.

Em um dos primeiros estudos encontrados na literatura sobre o assunto ${ }^{15}$, avaliou-se o deslocamento do centro de pressão corporal (COP) através da plataforma de força, e verificou-se que nos pacientes com sintomas motores mais graves, o COP apresentou-se deslocado anteriormente. O COP é uma variável importante associada à marcha e ao equilíbrio, e pode sofrer interferência de processos patológicos, sendo que deslocamentos extremos provavelmente aumentam o risco de queda nestes indivíduos e demandam atenção especial ao tratamento fisioterapêutico destes.

A necessidade de investigar proposiçóes de tratamentos eficazes de treinamento de marcha para pacientes com DP também foi encontrada na literatura em muitos estudos, especialmente através de análises cinemáticas $^{2,7,16}$. Estas análises permitem avaliar, por exemplo, variáveis como comprimento da passada e cadência, que são fortemente afetadas pela DP.

O comprimento da passada pode ser avaliado através de palmilhas pressurizadas, como encontrou-se em um estudo ${ }^{2}$ com 16 pacientes com DP e 16 sujeitos de um grupo controle, onde o principal achado foi que os pacientes com DP podem gerar um comprimento de passada normal quando são adicionadas informaçôes visuais ao treinamento ou quando são reforçadas estratégias 
de atenção. Relatou-se, também, que a manutenção dos ganhos na marcha pode durar por até duas horas após o treinamento, sugerindo que estas estratégias podem melhorar significativamente a marcha destes indivíduos, através de treinamentos prolongados ${ }^{2}$.

A cadência da passada está frequentemente relacionada à regulação do comprimento da passada. Anormalidades nesta relação surgem em decorrência de um defeito na capacidade de dimensionar o tamanho do passo, como consequência da contribuição deficitária dos gânglios da base para enviar informaçóes ao córtex motor ${ }^{16}$. Em investigação ${ }^{16}$ enfatizando esta relação antes e após a ingestão de levodopa, também através de palmilhas pressurizadas, em 20 pacientes e 20 sujeitos controle, mostrou-se que os pacientes foram capazes de aumentar o comprimento do passo em quantidades similares ao aumento da cadência após a ingestão de levodopa, e que este medicamento aumentou a amplitude da relação comprimento-cadência.

Em um dos primeiros estudos de análise tridimensional da marcha em pacientes com $\mathrm{DP}^{7}$, restriçóes nos parâmetros cinéticos, cinemáticos e espaço-temporais da marcha foram pesquisados simultaneamente, através do relato de caso com manipulação sistemática da levodopa em um sujeito idoso do sexo feminino, em estágio moderado de incapacidade funcional segundo escala UPDRS (Unified Parkinson's Disease Rating Scale). Os resultados dos dados espaço-temporais mostraram as características típicas de passos curtos e velocidade mais lenta ao final da dose da medicação dopaminérgica, bem como redução na amplitude total de movimento, sendo que estes parâmetros melhoraram durante o pico de ação do medicamento.

Quanto às avaliaçóes cinéticas do movimento na DP, a baropodometria tem ganhado destaque nos últimos anos, através de mensurações de variáveis como picos de pressóes plantares e oscilação do $\mathrm{COP}^{3-5}$, que traduzem a ação de forças internas e externas atuando sobre o corpo.

A baropodometria aplicada a sujeitos com DP mostrou-se confiável para distinguir os perfis de distribuição das pressóes plantares durante a marcha, revelando-se em concordância com a classificação estabelecida pelo escore de UPDRS5.

A distribuição das pressões plantares foi avaliada ${ }^{3}$ em pessoas com DP através do sistema PDG (pododyno- graph system), que consiste em duas palmilhas sensitivas, onde caminharam ao longo de uma trajetória de seis metros, em uma velocidade confortável auto-selecionada. $\mathrm{O}$ achado mais importante foi um acréscimo do pico de pressão na região do médio-pé de ambos os pés. Pacientes com DP caminharam com $66 \%$ da velocidade média, e $70 \%$ do comprimento da passada, comparativamente ao grupo controle. As fases de suporte e de duplo apoio da marcha foram significativamente prolongadas, enquanto a fase de balanço apresentou redução durante o ciclo da marcha.

Em estudo posterior ${ }^{4}$, utilizou-se o Sistema Pedar (Novel GmbH, Alemanha), composto por palmilhas pressurizadas, para determinar a distribuição da pressão plantar em pacientes com DP. Os pacientes mostraram uma redução significativa no pico de pressão da regiâo lateral do calcanhar comparativamente ao grupo controle, e maior distribuição de cargas para a região do médio-pé, comparativamente ao grupo controle. Náo obstante, parece que os pacientes com DP tem tendência a transferir as cargas dos calcanhares para as regióes do médio-pé e antepé. Os dados cinéticos da marcha foram relacionados com a classificação clínica da doença segundo o Webster Escore, indicando que quanto maior a diminuição nos picos de pressão na região dos calcanhares, mais severa é a manifestação da doença. Os resultados mostraram que mesmo pacientes em estágios leves e moderados da DP apresentam alteraçóes significativas na pisada. Os autores inferem que estes mecanismos provavelmente servem para compensar desequilíbrios durante a marcha.

Assim como nos dados cinemáticos, encontrou-se influência da ação da levodopa nos dados de pressóes plantares, em estudo ${ }^{5}$ comparando o comportamento da marcha nas fases on e off do tratamento medicamentoso através de palmilhas pressurizadas. Observou-se valores de pressóes plantares significativamente elevados na região do médio-pé durante a fase off, bem como na cabeça do segundo e terceiro metatarso 5 .

Corroborando com o estudo anterior ${ }^{5}$, o achado de valores de pressões plantares elevados na região do médio-pé em pacientes com DP também foi encontrado em outro estudo ${ }^{17}$ em 13 pacientes comparativamente a um grupo de 16 pessoas saudáveis, através do Sistema Gait View, que mostrou também, aumento significativo dos 
picos de pressóes na cabeça do quinto metatarso entre os pacientes. A avaliação dinâmica mostrou que a linha do COP dos pacientes está mais localizada na regiáo do antepé, com pouca ou nenhuma atuação no calcanhar.

Os aspectos biomecânicos da marcha na DP também vêm sendo avaliados após tratamentos com estimulação cerebral profunda (ECP), seja não-invasiva ou cirúrgica $^{10,12,17}$. A estimulação cerebral não-invasiva tem a propriedade de modular a atividade no córtex motor primário, e esta modulação está associada com a modulação das estruturas dos gânglios da base, mecanismo que poderia resultar em uma melhora da funçáo motora na $\mathrm{DP}^{18}$. Indivíduos com DP recebendo $\mathrm{ECP}$ não-invasiva do tipo estimulação magnética transcraniana repetitiva de $25 \mathrm{~Hz}$, por 8 sessóes, durante 4 semanas, mostraram melhora gradual na marcha e tarefas manuais, e esses benefícios permaneceram pelo menos 1 mês após o término do tratamento ${ }^{19}$.

Com relação ao tratamento cirúrgico da DP, estudos têm demonstrado que a ECP do núcleo subtalâmico (ECP-NST) está associada a melhores benefícios, com maior redução de medicação anti-parkisoniana do que a ECP do globo pálido (ECP-GP) ${ }^{20,21}$. A ECP-NST também melhora o tremor, a bradicinesia e a rigidez, além da melhora importante nos movimentos involuntários. Porém, a eficácia do tratamento é dependente da localização correta dos eletrodos ${ }^{10}$.

A estabilidade postural, uma habilidade física associada ao controle postural e importante para a execução segura de atividades diárias, foi avaliada através da área de oscilação do COP na posição estática, em indivíduos após ECP-NST ${ }^{22}$, revelando que a associação entre ECP-NST e a fase on do tratamento dopaminérgico mostrou os maiores ganhos na estabilidade postural.

A ECP-NST também mostrou-se capaz de provocar aumento no comprimento da passada e na velocidade da marcha, habilidades que normalmente apresentam-se com valores reduzidos em pacientes com DP, e este incremento foi maior quando associado ao tratamento dopaminérgico ${ }^{23}$.

O benefício da associação entre esses dois trata- mentos foi corroborado com relação ao aumento da velocidade da marcha, comprimento da passada e amplitude global de movimento, além da maior produção de potência nas articulaçóes do tornozelo e quadril ${ }^{24}$. Entretanto, há evidências que, em longo prazo, os ganhos na marcha com esses tratamentos diminuem devido à progressão da doença ${ }^{25}$.

A maioria das pesquisas incluídas nesta revisão apresenta convergência com relação aos dados espaciais e temporais da marcha na DP: velocidade diminuída, comprimento da passada menor, cadência aumentada - provavelmente como compensação à diminuição do comprimento da passada, e tempo de fase de apoio e duplo apoio aumentados.

Com relação aos dados de picos de pressão, houve consenso quanto à redução dos valores na regiáo dos calcanhares em geral e quanto ao aumento dos valores na região do médio-pé, o que pode estar relacionado ao desabamento do arco longitudinal medial. Quanto aos dados do COP, o principal achado foi a orientação mais medial do seu trajeto.

Nenhum dos estudos pesquisados avaliou a morfologia do pé em pacientes com DP. Alguns estudos relatam maior tendência a pisar com o pé plano, o que tem uma relaçấo positiva com a severidade da doença.

\section{CONCLUSÃO}

Conclui-se que muitas pesquisas vêm sendo desenvolvidas acerca da marcha na DP, com avanços importantes nesta área pelo advento de novas tecnologias de análises quantitativas da marcha. Dentre essas análises, a mais fortemente utilizada e explorada na DP, conforme as pesquisas aqui estudadas, é a cinemetria. As análises de distribuição da pressão plantar ainda apresentam resultados controversos e metodologias questionáveis. Por isso, novas pesquisas utilizando protocolos precisamente definidos e análise estatística bem aplicada, poderiam trazer muitos benefícios ao tratamento dos pacientes com DP. Como perspectivas de estudos futuros, há necessidade de relacionar-se a severidade da doença e o tipo de pé, além desta relação com a postura do paciente com DP. 


\section{REFERÊNCIAS}

1.Sofuwa O, Nieuwboer A, Desloovere K, Willems AM, Chavret F, Jonkers I. Quantitative gait analysis in Parkinson's disease: comparison with a healthy control group. Arch Phys Med Rehabil 2005;86:1007-13.

http://dx.doi.org/10.1016/j.apmr.2004.08.012

2.Morris M, Iansek R, Matyas T, Summers J. Stride length regulation in Parkinson's disease-Normalization strategies and underlying mechanisms. Brain 1996;119:551-68.

http://dx.doi.org/10.1093/brain/119.2.551

3.Nieuwboer A, De Weerdt W, Dom R, Peeraer L, Lesaffre E, Hilde F, et al. Plantar force distribution in parkinsonian gait: a comparison between patients and age-matched control subjects. Scand J Rehab Med 1999;31:185-92.

http://dx.doi.org/10.1080/003655099444533

4.Kimmeskamp S, Hennig E. Heel to toe motion characteristics in Parkinson patients during free walking. Clin Biomechan 2001;16:806-12.

http://dx.doi.org/10.1016/S0268-0033(01)00069-9

5.Pihet D, Moretto P, Defebvre L, Thevenon A. Analyse de la marche dans la maladie de Parkinson par enregistrement baropodométrique à l'aide de semelles embarquées. Revue Neurologique 2006;162:208-13.

http://dx.doi.org/10.1016/S0035-3787(06)75001-9

6.Okuno R, Fujimoto S, Akazawa J, Yokoe M, Sakoda S, Akazawa K. Analysis of spatial temporal plantar pressure pattern during gait in Parkinson's disease. 30th Annual International IEEE EMBS Conference Vancouver, British Columbia, Canada, August 2008; p20-4.

7.Morris M, McGinley J, Huxham F, Collier J, Iansek R. Constraints on the kinetic, kinematic and spatiotemporal parameters of gait in Parkinson's disease. Hum Mov Sci1999;18:461-83.

http://dx.doi.org/10.1016/S0167-9457(99)00020-2

8.Chung A, Lobb B, Nutt J, McNames J, Horak F. Objective measurement of dyskinesia in Parkinson's disease using a force plate. Mov Disord 2010;25:602-8. http://dx.doi.org/10.1002/mds.22856

9.Švehlík M, Zwick B, Steinwender G, Linhart WE, Schwingenschuh P, Katschnig P, et al. Gait Analysis in Patients With Parkinson's Disease Off Dopaminergic Therapy. Arc Phys Med Rehab 2009;90:1880-6.

http://dx.doi.org/10.1016/j.apmr.2009.06.017

10.Berney A, Vingerhoets F, Perrin A, Guex P, Villemure JG, Burkhard PR, et al. Effect on mood of subthalamic DBS for Parkinson's disease: a consecutive series of 24 patients. Neurology 2002;59:1427-9.

http://dx.doi.org/10.1212/01.WNL.0000032756.14298.18

11.Lubik S, Fogel W, Tronnier V, Krause M, König J, Jost WH. Gait analysis in patients with advanced Parkinson disease: different or additive effects on gait induced by levodopa and chronic STN stimulation. J Neural Transm 2006;113:163-73.

http://dx.doi.org/10.1007/s00702-005-0310-8

12.Ferrarin M, Rizzone M, Bergamasco B, Lanotte M, Recalcati M, Pedotti A, et al. Effects of bilateral subthalamic stimulation on gait kinematics and kinetics in Parkinson's disease. Exp Brain Res 2005;160:517-27.

http://dx.doi.org/10.1007/s00221-004-2036-5
13.Yang C-C. Gait cycle parameters recognition using the wearable motion detector. In: Yang CC. Development of a Home Telehealth System for Telemonitoring Physical activity and Mobility of the elderly. Gerontechnology Research Center, Yuan Ze University 2011; p.1-42.

14.Hughes J, Bowes S, Leeman A, O’Neill CJ, Deshmukh AA, Nicholson PW, et al. Parkinsonian abnormality of foot strike: a phenomenon of ageing and/ or one responsive to levodopa therapy? Br J Clin Pharmacol 1990;29:179-86. http://dx.doi.org/10.1111/j.1365-2125.1990.tb03617.x

15.Lee R, Tonolli I, Viallet F, Aurenty R, Massion J. Preparatory postural adjustments in parkinsonian patients with postural instability. Can J Neurol Sci 1995;22:126-35.

16.Morris M, Iansek R, Matyas T, Summers J. Abnormalities in the Stride Length-Cadence Relation in Parkinsonian Gait. Mov Dis 1998;13:61-9.

http://dx.doi.org/10.1002/mds.870130115

17.Chen X, Wang C, Liu H, Ding M, Lin M, Huang D. Study of plantar pressure in Parkinson disease patients. Parkin Relat Dis 2012;81-159.

18.Fregni F, Pascual-Leone A. Technology Insight: noninvasive brain stimulation in neurology: perspectives on the therapeutic potential of rTMS and tDCS. Nat Clin Pract 2007;3:383-93.

http://dx.doi.org/10.1038/ncpneuro0530

19.Miranda PC, Lomarev M, Hallett M. Modeling the current distribution during transcranial direct current stimulation. Clin Neurophysiol 2006;117:1623-29.

http://dx.doi.org/10.1016/j.clinph.2006.04.009

20.Vesper J, Klostermann F, Stockhammer F, Funk T, Brock M. Results of chronic subthalamic nucleus stimulation for Parkinson's disease: a 1-year follow-up study. Surg Neurol 2002;57306-11.

21.Devos D, Labyt E, Derambure P, Bourriez JL, Cassim F, Reyns N, et al. Subthalamic nucleus stimulation modulates motor cortex oscillatory activity in Parkinson's disease. Brain 2004;127:408-19.

http://dx.doi.org/10.1093/brain/awh053

22.Maurer C, Mergner T, Xie J, Faist M, Lucking CH. Effect of chronic bilateral subthalamic nucleus (STN) stimulation on postural control in Parkinson's disease. Brain 2003;126:1146-63.

http://dx.doi.org/10.1093/brain/awg100

23. Faist M, Xie J, Kurz D, Berger W, Maurer C, Pollak P, Lücking CH. Effect of bilateral subthalamic nucleus stimulation on gait in Parkinson's disease. Brain 2001;124:1590-600.

http://dx.doi.org/10.1093/brain/124.8.1590

24.Ferrarin M, Rizzone M, Bergamasco B, Lanotte M, Recalcati M, Pedotti A, et al. Effects of bilateral subthalamic stimulation on gait kinematics and kinetics in Parkinson's disease. Exp Brain Res 2005;160:517-27.

http://dx.doi.org/10.1007/s00221-004-2036-5

25.Fasano A, Romito L, Daniele A, Piano C, Zinno M, Bentivoglio AR, et al. Motor and cognitive outcome in patientswith Parkinson's disease 8 years after subthalamic implants. Brain 2010;133:2664-76.

http://dx.doi.org/10.1093/brain/awq221 